\title{
Nanoparticle floating-gate transistor memory based on solution- processed ambipolar organic semiconductor
}

\author{
Sheng Sun ${ }^{1,2}$, and Shengdong Zhang ${ }^{1, *}$ \\ ${ }^{1}$ School of Electronic and Computer Engineering, Peking University Shenzhen Graduate School, Peking University, Shenzhen, China \\ ${ }^{2}$ TCL China Star Optoelectronics Technology Co., LTD. Shenzhen, China
}

\begin{abstract}
Organic thin-film transistor memory based on nano-floating-gate nonvolatile memory was demonstrated by a simple method. The gold nanoparticle that fabricated by thermally evaporated acted as the floating gate. Spin coated PMMA film acted as the tunneling layer. A solution-processed ambipolar semiconductor acted as the active layer. Because of the existence of both hole and electron carriers in bipolar semiconductor materials, it is more conducive to the editing and erasing of memories under positive and negative pressure. The memory based on metal nanoparticles and organic bipolar semiconductor shows good read-write function.
\end{abstract}

\section{Introduction}

Memory is one of the main applications of organic thin film transistors. In the 1970s, Frohman-Bentchkowsky et al. prepared floating gate transistors, which realized the injection and storage of charges in the floating gate, demonstrating the feasibility of using this structure as a memory.[1] In the following decades, transistor memory has made great progress and gradually moved towards practical applications. With the research and development of organic semiconductors, the first organic transistor memory appeared in 1986. It was prepared by Tsumura et al. with polythiophene as the active layer. [2] Since then, this field has made considerable progress. In 2009, Sekitani et al. reported the first flexible memory organic thin film transistor memory, and the write voltage was lower than $10 \mathrm{~V}$.[3] The device is prepared on a polyethylene-2,6-naphthalate (PEN) substrate and adopts a floating gate structure with aluminum oxide as the insulating layer gate, and $20 \mathrm{~nm}$ thick aluminum film as the floating gate layer. When a certain voltage is applied, the charge will be transferred to the floating gate layer by means of thermal excitation and quantum tunneling to achieve storage.

Generally, organic thin film transistor memories are divided into the following three categories according to different floating gate structures: ferroelectric polymer transistor memories, electret polymer transistor memories, and nanoparticle floating gate transistor memories. The storage medium of the ferroelectric polymer transistor memory is a ferroelectric polymer. The working principle is that when the gate voltage changes, the polarity of the ferroelectric polymer will change, and then the current can reflect the two stable states of on and off to storage information. Electret polymer transistor memory uses the defects of the electret polymer itself and other properties. Under a certain gate voltage, the charge of the organic semiconductor is injected into the insulating layer and maintains a stable state, and the electret that retains the charge is polymerized with the non-injected electret. The memory will reflect different currents under the same voltage to realize information storage. The working principle of the nanoparticle floating gate transistor memory is similar to electret polymer transistor memory, the difference is that the floating gate nanoparticles replace the electret to store charge.

In recent years, the research of transistor memory based on nanoparticle floating gate structure has made important progress.[4-6] Metal nanoparticles, such as aluminum, copper, silver, and gold, are often used as nano-floating-gates. Kung-Hwa Wei et al. have done a systematic study on it, and the study shows that the size of the hole and electron injection barrier is closely related to the work function of the metal electrode.[7] Among many metal materials, gold is very suitable as a storage medium in terms of chemical stability and metal work function.

Compared with inorganic transistor memory, due to the characteristics of organic materials, organic transistor memory has many advantages: lightness, flexibility, solution processing, and suitability for large-area preparation. Therefore, organic transistor memory has a broader application prospect. For example, organic transistor memory can be fabricated on a flexible substrate to achieve flexible storage.[8-11] Using solution processing or even inkjet printing processes, organic transistor memory can be fabricated in a large area, greatly reducing costs. However, organic transistor memory still has some problems, such as poor stability, high operating voltage, unsatisfactory retention, long programming time, and further clarification of the

\footnotetext{
* Corresponding author: zhangsd@,pku.edu.cn
} 
working mechanism. There are many problems that need to be resolved.

In this paper, an organic transistor memory based on a nanoparticle suspended gate structure is prepared. Gold nanoparticles are used as a floating gate, polymethyl methacrylate (PMMA) is used as a tunnel insulating layer, and a bipolar organic semiconductor material is used as an active layer to prepare a nanoparticle suspension gate. In organic transistor memory, due to the presence of both holes and electron carriers in bipolar materials, the injection of different types of carriers under different voltages is realized, and the storage window is increased.

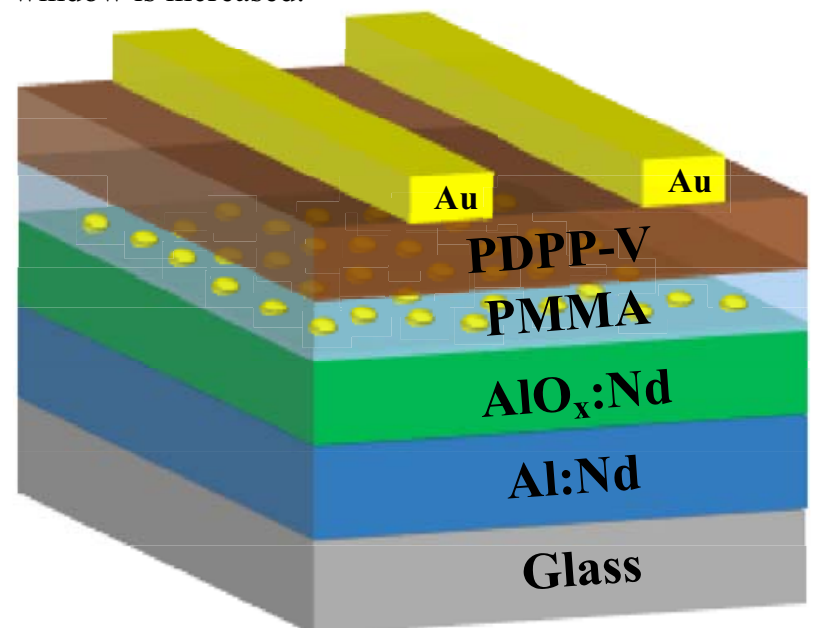

Fig. 1. Diagrammatic sketch of nano floating gate organic transistor

\section{Experimental Details}

Substrate cleaning: Put the glass substrate in acetone, isopropanol, lotion, deionized water, and isopropanol in the order of ultrasonic cleaning for 10 minutes, and put it in an oven at $80^{\circ} \mathrm{C}$ for drying.

Preparation of gate electrode and gate insulating layer: Sputter Al-Nd alloy film, and anodize to form $\mathrm{AlO}_{\mathrm{x}}: \mathrm{Nd}$ gate insulating layer.

Preparation of gold particle nano-floating grid: the above-mentioned substrate is turned upside down on the metal mask, placed in a vacuum evaporation equipment, and then vacuumed to below $3 \times 10^{-4} \mathrm{~Pa}$, then $0.1 \AA \cdot \mathrm{s}^{-1}$ The evaporation rate is $1 \mathrm{~nm}$ gold as the nano floating gate.

The preparation of the tunnel insulation layer: Take the above-mentioned substrate out of the equipment, spin-coating PMMA chlorobenzene solution, concentration $30 \mathrm{mg} / \mathrm{mL}$, rotating speed $5000 \mathrm{rpm}$, spin coating $30 \mathrm{~s}$, and then continue annealing in a glove box at $200^{\circ} \mathrm{C}$ for $30 \mathrm{~min}$, and it can get $100 \mathrm{~nm}$ thick PMMA film.

Preparation of the active layer: Prepare a PDPP-V solution with a solvent of dichlorobenzene and a concentration of $0.5 \mathrm{mg} / \mathrm{mL}$. Conduct spin-coating on the above substrate at $2000 \mathrm{rpm}$ in a nitrogen glove box. And then continue annealing in a glove box at $100{ }^{\circ} \mathrm{C}$ for $30 \mathrm{~min}$, and finally a $30 \mathrm{~nm}$ thick film is formed.
Preparation of source and drain electrodes: Place the above-mentioned substrate upside down on the metal mask, put it into the vacuum evaporation equipment, evacuate to below $3 \times 10^{-4} \mathrm{~Pa}$, and then use $0.1 \sim 0.3$ $\AA \cdot \mathrm{s}^{-1}$ The evaporation rate is $45 \mathrm{~nm}$ gold as the source and drain electrodes, and the aspect ratio is $500 \mu \mathrm{m}: 70$ $\mu \mathrm{m}$.

Device electrical performance test: It is tested by the semiconductor parameter tester Agilent $4155 \mathrm{C}$ at room temperature in the air.

\section{Results and Discussion}

As shown in our earlier work, FBT-Th $_{4}(1,4)$ is an organic semiconductor with high hole mobility.[12] Fig. 2 shows the OTFT memory with FBT-Th $4(1,4)$ as the active layer. It can be seen that after writing to a certain negative pressure, the holes in the channel will tunnel through the tunnel insulation and enter the floating gate of metal nanoparticles, thus generating an internal bond electric field opposite to the gate voltage direction, resulting in a negative drift of the threshold voltage. Unfortunately, when the positive gate voltage is applied, the transfer curve does not deviate to the positive gate voltage direction, indicating that the holes stored in the floating gate cannot be erased by positive gate voltage. This is also a problem encountered by many unipolar memories, some of which are erased by laser irradiation.

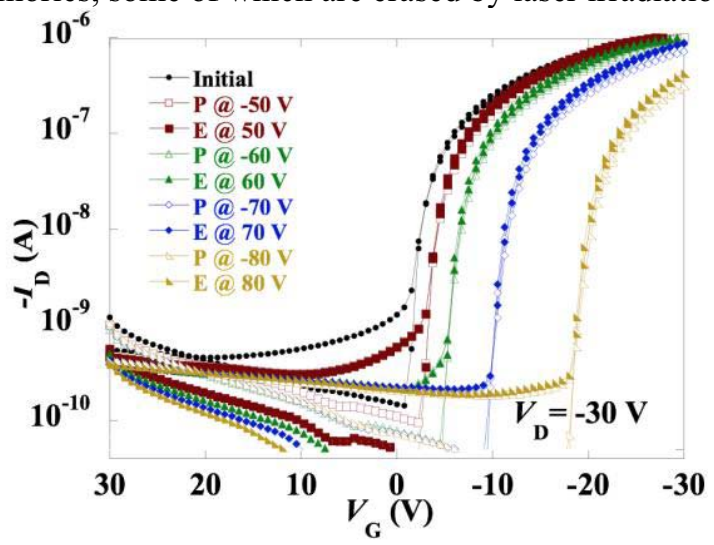

Fig. 2. Transfer curves of the organic memory devices with unipolar semiconductor according to the different programming/erasing biases at drain bias of $-30 \mathrm{~V}$.

PDPP-V is a bipolar organic semiconductor material. We have adopted a top-gate low-contact structure to obtain the best performance of the device. The device structure diagram, molecular formula and corresponding transfer characteristic curve are shown in Fig. 3. The hole mobility of the device is $0.15 \mathrm{~cm}^{2} \mathrm{~V}^{-1} \mathrm{~s}^{-1}$, and the electron mobility is $0.08 \mathrm{~cm}^{2} \mathrm{~V}^{-1} \mathrm{~s}^{-1}$. The IDVG curve shows that PDPP-V exhibits obvious bipolar characteristics. 
(a)

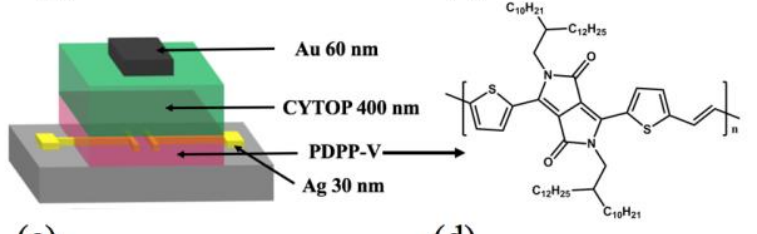

(c)

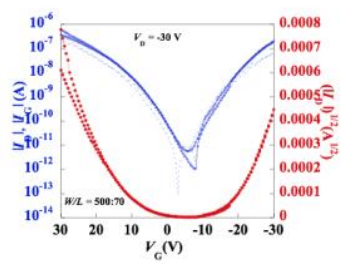

(d)

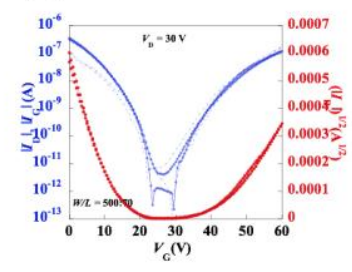

Fig. 3. (a) Schematic diagram of PDPP-V OTFT, (b) chemical structures of PDPP-V, transfer characteristics : (c) $V_{\mathrm{D}}=-30 \mathrm{~V}$ and (d) $V_{\mathrm{D}}=30 \mathrm{~V}$.

Fig. 4 shows the OTFT memory with PDPP-V organic bipolar semiconductor material as the active layer. When a certain negative pressure is applied for the write operation, similarly, the holes in the channel are injected into the metal nanoparticle floating gate, which eventually causes the transfer curve to shift to the negative gate voltage direction. When the positive gate voltage is applied, the transfer curve to the direction of the positive gate voltage is shifted, which means that because PDPP-V is a bipolar material, electron carriers also exist in the channel. Under the action of the positive gate voltage, the electrons in the channel will be injected into the metal nano-floating gate. The holes will be neutralized and electrons are stored in the floating gate, which eventually causes the transfer curve to shift toward the positive gate voltage. The bipolar materials can not only perform write and erase operations more easily, but also theoretically form a larger storage window, and under different programming voltages, different storage windows appear, reflecting certain multi-level storage. When the programming voltage value is $80 \mathrm{~V}$, the storage window reaches $20 \mathrm{~V}$.

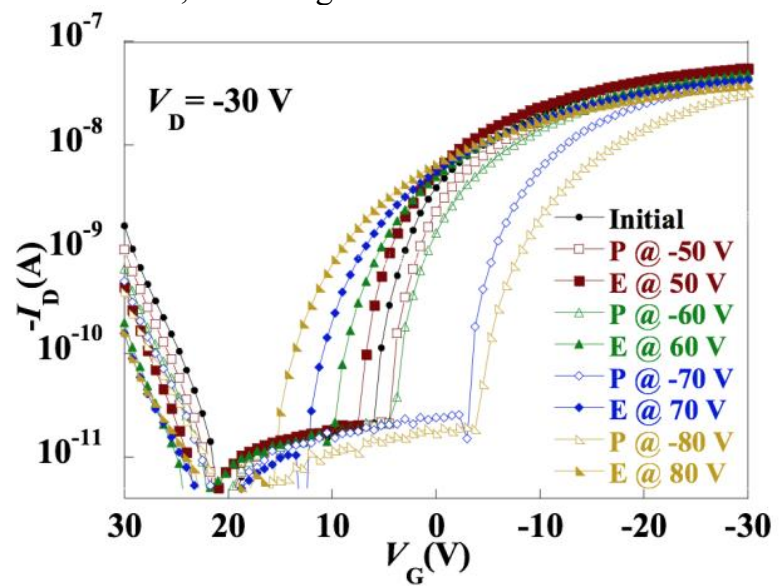

Fig. 4. Transfer curves of the organic ambipolar memory devices according to the different programming/erasing biases at drain bias of $-30 \mathrm{~V}$

Fig. 5 is the retention characteristic curve of the device. According to the programming transfer characteristic curve of the memory in Fig. 4, we select $V_{\mathrm{G}}=80 \mathrm{~V}$ after erasing, and the $I_{\mathrm{D}}$ under the state of $V_{\mathrm{D}}$ $=-30 \mathrm{~V}$ and $V_{\mathrm{G}}=0 \mathrm{~V}$ is on, and be tested every $20 \mathrm{~s}$ after erasing. After selecting $V_{\mathrm{G}}=-80 \mathrm{~V}$ for programming, the $I_{\mathrm{D}}$ under $V_{\mathrm{D}}=-30 \mathrm{~V}, V_{\mathrm{G}}=0 \mathrm{~V}$ is off state, and the test is performed every $20 \mathrm{~s}$ after programming. The results are as shown in the Fig. 5, the device can still maintain a switching ratio of more than 100 after $1000 \mathrm{~s}$.

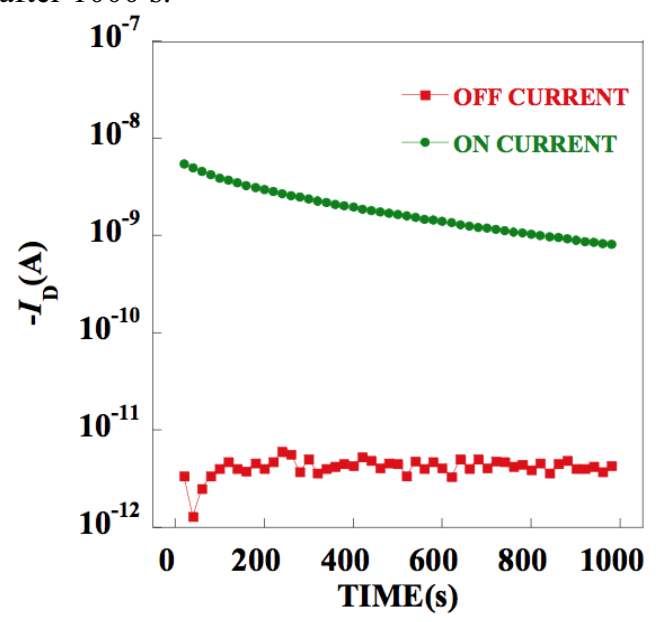

Fig. 5. The retention characteristic curve of the nanoparticle floating-gate ambipolar transistor memory.

Fig. 6 shows the programming/reading/erasing /reading $(\mathrm{P} / \mathrm{R} / \mathrm{E} / \mathrm{R})$ endurance of the nanoparticle floating-gate ambipolar transistor memory, at $V_{\mathrm{D}}$ of -30 $\mathrm{V}$ with $V_{\mathrm{G}}=-80 \mathrm{~V} / 0 \mathrm{~V} / 80 \mathrm{~V} / 0 \mathrm{~V}$ respectively. The bipolar organic memory has obvious nonvolatile storage function, as shown in Fig. 6. This work provides a simple method for fabrication of nonvolatile memory.

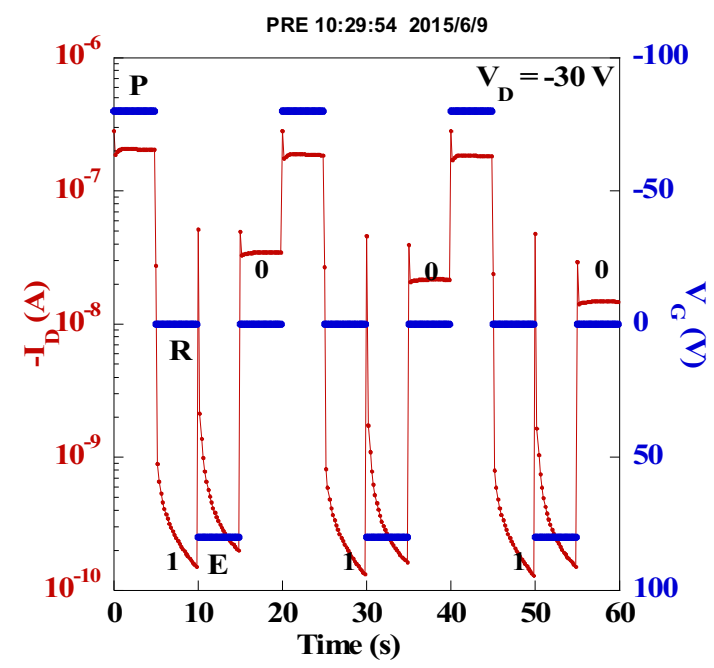

Fig. 6. The storage cycles of the organic ambipolar memory devices with $V_{\mathrm{G}}=-80 \mathrm{~V}, 0 \mathrm{~V}$ and $80 \mathrm{~V}$ for programming, reading, and erasing, respectively at drain bias of $-30 \mathrm{~V}$.

\section{Conclusion}

In this paper, the nonvolatile memory was prepared by evaporating Au nanoparticles as floating gate, PMMA as tunneling insulator, and PDPP-V as active layer. 
Because bipolar organic semiconductors have both holes and electron carriers, the programming and erasing operations can be realized by gate voltage, and the multilevel memory can be realized under different gate voltage programming. Although the performance of the device is not outstanding, the whole process is simple, and the programming and erasing operation can be realized only by adjusting the gate voltage, which reflects the application potential of organic thin film transistor in organic memory.

\section{References}

1. D. Frohman-Bentchkowsky, M. Lenzlinger, J. Appl. Phys. 40, 8(1969)

2. A. Tsumura, H. Koezuka, T. Ando, Appl. Phys. Lett. 49, 18(1986)

3. T. Sekitani, T. Yokota, U. Zschieschang, H. Klauk, S. Bauer, K. Takeuchi, M. Takamiya, T. Sakurai, T. Someya, 326(2009)

4. D. Lee, Y. Choi, E. Hwang, M. S. Kang, S. Leead J. H. Cho, Nanoscale, 8, 17(2016)

5. X. J. She, C. H. Liu, Q. J. Sun, X. Gao, S. D. Wang, Org. Electron. 13, 10(2012)

6. K. J. Baeg, Y. Y. Noh, H. Sirringhaus, D.T. Kim, Adv. Func. Mater. 20, 2(2010)

7. Chen C M, Liu C M, Wei K H, et al. C. M. Chen, C. M. Liu, K. H. Wei, U. S. Jeng, C. H. Sub, J. Mater. Chem. 22, 2(2012)

8. S. T. Han, Y. Zhou, Z. X. Xu, V. A. L. Roy, T. F. Hung, J. Mater. Chem. 21, 38(2011)

9. W. Wang, D. G. Ma, Appl. Phys. Lett. 96(2010)

10. W. W., Sun, K. Hwang, K. L. Kim, J. H. Lee, S. M. Cho, C. Park, ACS Appl. Mater. Interfaces, 7, 20(2015)

11. J. Ying, J. H. Han, L. Y. Xiang, W. Wang, W. F. Xie, Current Appl. Phys. 15(2015)

12. S. Sun, L. F. Lan, P. Xiao, Z. H. Chen, Z. G. Lin, Y. Z. Li, H. Xu, M. Xu, J. W. Chen, J. B. Peng, Y. Cao, J. Mater. Chem. C. 3(2015) 